44th AIAA/ASME/ASCE/ASC Structures, Structural Dynamics, and Materials Conference, Norfolk, VA, 7-10 April 2003

\title{
NONLINEAR TIME DELAYED FEEDBACK CONTROL OF AEROELASTIC SYSTEMS: A FUNCTIONAL APPROACH
}

\author{
Piergiovanni Marzocca ${ }^{*}$, Liviu Librescu ${ }^{\dagger}$ and Walter A. Silva \\ ${ }^{*}$ Virginia Polytechnic Institute and State University, Blacksburg, VA 24061-0219, \\ ${ }^{\ddagger}$ NASA Langley Research Center, Hampton, VA 23681-2199.
}

\begin{abstract}
In addition to its intrinsic practical importance, nonlinear time delayed feedback control applied to lifting surfaces can result in interesting aeroelastic behaviors. In this paper, nonlinear aeroelastic response to external time-dependent loads and stability boundary for actively controlled lifting surfaces, in an incompressible flow field, are considered. The structural model and the unsteady aerodynamics are considered linear. The implications of the presence of time delays in the linear/nonlinear feedback control and of geometrical parameters on the aeroelasticity of lifting surfaces are analyzed and conclusions on their implications are highlighted.
\end{abstract}

\section{NOMENCLATURE}

a Dimensionless elastic axis position measured from the mid-chord, positive aft

$b \quad$ Semi-chord

$c, k$ Damping and stiffness parameters of 1-DOF plunging airfoil, respectively

$c_{h}, c_{\alpha}, c_{\beta}$ Damping parameters in plunging, pitching and flapping, respectively

$C(s)$ Theodorsen's function

$C_{L \alpha} \quad$ Lift-curve slope, $2 \pi$

$e \quad$ Dimensionless leading edge flap position measured from the mid-chord, positive aft

$\mathbf{F}_{\mathbf{a}}, \mathbf{F}_{\mathbf{b}}$ Aerodynamic and time-dependent load vectors

$\mathbf{F}_{\mathbf{c}} \quad$ Control force

$\mathbf{g}_{\mathbf{p}}, \mathbf{g}_{\mathbf{v}}, \mathbf{g}_{\mathbf{n c}}$ Proportional, velocity and nonlinear feedback control gains matrix

$g_{p}, g_{v}, g_{n c} ; \mathbf{g}_{p}, \mathbf{g}_{v}, \mathbf{g}_{n c}$ Proportional, velocity and nonlinear feedback control gains of 1-DOF airfoil, and their dimensionless counterparts, respectively

G Control input matrix

* Visiting Assistant Professor, Department of Engineering Science and Mechanics, Member AIAA.

$\dagger$ Professor of Aeronautical and Mechanical Engineering, Department of Engineering Science and Mechanics.

* Senior Research Scientist, Senior Aerospace Engineer, Aeroelasticity Branch, Structures and Materials Competency, Senior Member AIAA. $h, \alpha, \beta$ Plunging, pitching and flap displacements, respectively

$I_{\alpha}, I_{\beta}$ Mass moment of inertia per unit length of the wing-flap and of the flap about the elastic axis, and about the flap axis of rotation, respectively

$k_{h}, k_{\alpha}, k_{\beta}$ Stiffness parameters in plunging; torsional stiffnesses of the wing and flap about the elastic axis, and about the flap axis of rotation, respectively

$\bar{k} \quad$ Reduced frequency, $\omega b / U_{\infty}$

$L_{a}, L_{b}, L_{c}$ Aerodynamic lift, time-dependent external load, active feedback control, respectively

$m, \mu$ Mass of the wing per unit of length and mass ratio, $\left(\equiv m / 2 \rho_{\infty} b^{2}\right)$, respectively

$\mathbf{M}, \mathbf{K}, \mathbf{B}$ Structural matrices,

$\mathbf{M}_{\mathbf{a}}, \mathbf{K}_{\mathbf{a}}, \mathbf{B}_{\mathbf{a}}$ Aerodynamic matrices

$r_{\alpha}, r_{\beta}$ Dimensionless radii of gyration of the wing-flap, $\left(I_{\alpha} / m b^{2}\right)^{1 / 2}$, and of the flap, $\left(I_{\beta} / m b^{2}\right)^{1 / 2}$, respectively

$s \quad$ Laplace transform variable

$S_{\alpha}, \chi_{\alpha}$ Static unbalance about the elastic axis and its dimensionless counterpart, $S_{\alpha} / m b$

$S_{\beta}, \chi_{\beta}$ Static unbalance about the flap axis of rotation and its dimensionless counterpart, $S_{\beta} / m b$

$t, \sigma ; \tau$ Time and dummy time variables, respectively; dimensionless time variable, $t U_{\infty} / b$

$T_{i} \quad$ Theodorsen's constants

$U_{\infty}, V$ Freestream speed and its dimensionless counterpart $V=U_{\infty} / \omega_{0} b$, respectively.

$\mathbf{x} \quad$ Plunging, pitching and flap displacement vector $\zeta_{\xi}, \zeta_{\alpha}, \zeta_{\beta}$ Structural damping ratios in plunging $\left(\equiv c_{h} / 2 m \omega_{h}\right)$, pitching $\left(\equiv c_{\alpha} / 2 I_{\alpha} \omega_{\alpha}\right)$, and flapping $\left(\equiv c_{\beta} / 2 I_{\beta} \omega_{\beta}\right)$, respectively

$\xi, \alpha$ Plunging and pitching displacement quantities

$\rho_{\infty} \quad$ Air density

$t_{i}, \widetilde{\tau}_{i}$ Time delay and dimensionless time delays, $t_{i} U_{\infty} / b, i=\overline{1,4}$, respectively 
44th AIAA/ASME/ASCE/ASC Structures, Structural Dynamics, and Materials Conference, Norfolk, VA, 7-10 April 2003

$\phi(\tau)$ Wagner's function

$\omega_{h}, \omega_{\alpha}, \omega_{\beta}$ Uncoupled frequencies in plunging,

$$
\left(k_{h} / m\right)^{1 / 2} \text {, pitching, }\left(k_{\alpha} / I_{\alpha}\right)^{1 / 2} \text {, and flapping }
$$$$
\left(k_{\beta} / I_{\beta}\right)^{1 / 2} \text {, respectively }
$$

\section{$\underline{\text { INTRODUCTION }}$}

$\mathcal{P}$ ast and recent literature on aeroelasticity is mostly devoted to linear models and to harmonic solutions. Often experimental results are interpreted by assuming a linear behavior of the physical model. Recently, special emphasis has been placed on the role of nonlinearities on aeroelastic instabilities, and for the most part, these studies have focused on the qualitative nonlinear behavior of open loop aeroelastic systems. Still, it is rather accurate to say that currently there is a lack of research work on closed loop dynamics of aeroelastic systems. The nonlinear aspects that we are addressing in this research are those which arise in the description of the feedback delayed control.

This study can lead to new qualitative results in the areas of flutter instability boundary and aeroelastic response to gust and blast loadings.

As a bottom line, it is imperative that the occurrence of flutter phenomena be suppressed, as to avoid the catastrophic failure of the structure [1]. These facts emphasize the importance of developing proper methodologies for the active control of structural systems, enabling one to raise the flutter speed, to enhance the aeroelastic response (attenuating excessive vibrations), and convert the unstable LCO, in which case the flutter boundary is catastrophic, into a stable $\mathrm{LCO}$, for which case the flutter boundary is benign.

The determination of the stability boundary of linear/nonlinear actively controlled aeroelastic systems, where the presence of the unavoidable time delays between controller and actuators is included, constitutes an important practical problem. In fact, the actuators may input energy at the exact moment when the controlled system does not need it [2-4]. These delays can be very detrimental in the sense of deteriorating the control performance and can even cause irregular motions, producing instability of the aeroelastic system. However, there are cases where those delays are used to control chaotic motions [5].

For a better understanding of the challenging problem related to the nonlinear delayed feedback control, the model of a 2-D wing section was considered and its effect on the aeroelastic response and flutter has been investigated.

The methodology used in this work is based on Volterra series and indicial functions in conjunction with a feedback control [1]. Volterra's functional series technique was proven to be an efficient tool in the solution of various nonlinear aeroelastic problems [4]. The Volterra series approach can also be used toward the formulation of the stability criteria for systems featuring time delays.

\section{BACKGROUND}

The problem of controlling unstable motion is an important subject in aeroelasticity. The renewed interest of aeroelasticians in this subject started, however, with the observation that a large number of unstable periodic orbits, (limit cycle oscillations LCO), embedded in chaotic attractors can be stabilized by weak external forces.

Two main methods for controlling unstable motions have been established. The first one was developed by Ott, Grebogi, and Yorke [6]. This method, based on the invariant manifold structure of unstable orbits, is theoretically well understood, but since it is difficult to apply to fast experimental systems, it has a limited interest. Another approach is due to Pyragas [7]. This method uses time-delayed controlling forces; it can easily be applied to real experimental situations, but so far the control mechanism has been poorly understood from a theoretical point of view. Considerable research has been done for more than three decades on various aspects of dynamical systems with delayed factors in the state variables and/or control inputs [8-12].

Various stability criteria and numerical approaches have been presented in recent years, see [13-14] and references cited therein. Consequently, time-delayed feedback has been applied widely [3,15-17]. In Ref. [8] an investigation of the stability and chaos for wheel suspensions was presented.

For aeroelastic systems in Ref. [5] the time-delayed feedback was applied toward the control of the chaotic motion of a 2-D lifting surface, with cubic pitching stiffness and linear viscous damping, using the feedback control method of Pyragas [7].

Stability analysis has been conducted in Ref. [2] for a linear, damped SDOF system with time delays in the displacement and in the velocity feedback.

With the exception of [5], the use of time-delayed feedback in aeroelasticity has been very limited. This is due to the fact that the characteristic equation of the delayed system is transcendental, i.e. has infinite number of roots, so it is neither possible to solve for its roots, nor to easily find approximate solutions $[2,12]$. Moreover, the aeroservoelastic problem is extremely complex. For this reason, as a first step toward the nonlinear analysis, the stability of linear differentialdifference aeroelastic equations has to be studied [3].

The present study can provide broad information and answer some basic questions, such as whether the aeroelastic stability is affected by the presence of delays that appear in the feedback, and whether the system 
stability is robust with respect to small variations of the feedback gains.

As reported in [4], multiple degree-of-freedom aeroelastic systems, including structural and aerodynamic nonlinearities, can be investigated via a combined Volterra series $[18,19]$ and Indicial functions technique $[1,4]$. Originally, the methods of Volterra series and Volterra kernel identification were developed to identify the nonlinear behavior in electrical circuits [19]. In [1] Volterra's series approach has been applied to the open/closed-loop aeroelasticity of airfoils. It was shown that the method provides opportunities for developing a unified and efficient way to address problems of nonlinear aeroelasticity. By performing an analytical linear stability analysis of the aeroelastic system via the use of the first order Volterra kernel, it is determined which class of orbits is accessible to timedelayed feedback control methods. Explicit expressions for important quantities like the critical time-delays and control gains or the dependence of the transient behavior on the control parameters, are derived. In this paper the stability boundary of a reduced order open/ closed-loop aeroelastic system incorporating a nonlinear time delayed feedback control is presented. The goals of designing such a system consist of controlling the aeroelastic response behavior, increasing the flutter speed and converting the catastrophic flutter boundary into a benign one.

\section{ANALYTICAL DEVELOPMENTS}

The first step towards the modeling of an open/closedloop aeroelastic system with nonlinear time delayed feedback control via the Volterra series approach is to determine the aeroelastic kernels. For the purpose of the present analysis, the approach presented in Refs. [1,4] has been modified. The basic assumptions and a detailed procedure are presented in Ref. [4]. For exhaustive treatments of the Volterra series concept applied in structural dynamics, the interested reader is referred to Ref. [20].

The aeroelastic kernels including control effects are derived in terms of the structural parameters, unsteady aerodynamics, proportional (PFC) and velocity (VFC) feedback control gains and feedback delays. Based on these kernels, the time histories and flutter boundary of the open/closed loop delayed aeroelastic system are obtained. The determination for each specific flight condition of the corresponding linear and nonlinear kernels of the Volterra series is required [4]. The open/closed-loop aeroelastic governing equation of an airfoil featuring plunging-pitching-flap deflection motion and subjected to external time-dependent loads can be expressed as

$$
\mathbf{M}_{\mathbf{s}} \ddot{\mathbf{x}}(t)+\mathbf{B}_{\mathbf{s}} \dot{\mathbf{x}}(t)+\mathbf{K}_{\mathbf{s}} \mathbf{x}(t)=m^{-1}\left[\mathbf{F}_{\mathbf{a}}(t)+\mathbf{F}_{\mathbf{b}}(t)\right]+\mathbf{G u}(t)(1)
$$

where $\mathbf{x}(t)=[h(t), \alpha(t), \beta(t)]^{T}, \mathbf{u}(t)$ is the control input (for a 3-DOF, for example, a torque applied at the flap [21]). The unsteady aerodynamic loads are represented by

$$
\mathbf{F}_{\mathbf{a}}(t)=\mathbf{M}_{\mathbf{a}} \ddot{\mathbf{x}}(t)+\mathbf{B}_{\mathbf{a}} \dot{\mathbf{x}}(t)+\mathbf{K}_{\mathbf{a}} \mathbf{x}(t)+\mathbf{F}_{\mathbf{c}}(t)
$$

The significance of the other parameters is well known, see $[22,23]$. As a remark, a closed-loop system can be seen as an open-loop system where the transfer function includes the feedback control. In Eq. (1) the state feedback control with delay can be expressed in the form:

$$
\mathbf{G u}(t-\tau)=\mathbf{g}_{\mathbf{p}} \mathbf{x}(t-\tau)+\mathbf{g}_{\mathbf{v}} \dot{\mathbf{x}}(t-\tau)+\mathbf{g}_{\mathbf{n c}} \mathbf{x}^{3}(t-\tau)
$$

where $\mathbf{g}_{\mathbf{p}}, \mathbf{g}_{\mathbf{v}}, \mathbf{g}_{\mathbf{n c}}$ are the feedback gain matrices for the displacement, velocity, and the nonlinear term, respectively. Since the aeroelastic system incorporating feedback control forces and moments with time-delays in the state feedback is of an evident complexity, for a better understanding of the problem and of the present procedure, a simplified model has been adopted.

\section{DELAYED AEROELASTIC SYSTEM: STABILITY AND RESPONSE}

Some concepts related with the aeroelastic response and stability of the 1-DOF plunging airfoil in the presence of time delays between the sensing and the action of the actuator are presented next.

A 1-DOF plunging airfoil is modeled as [1,4]:

$$
m \ddot{h}(t)+\operatorname{ch}(t)+k h(t)=-L_{a}(t)+L_{b}(t)+L_{c}(t)
$$

In the right hand side of this equation, the unsteady aerodynamic lift is represented as

$$
L_{a}(t)=C_{L \alpha} \rho U_{\infty} b \int_{-\infty}^{t} \phi(t-\sigma) \frac{\partial \dot{h}(\sigma)}{\partial \sigma} d \sigma+\frac{1}{2} \rho C_{L \alpha} b^{2} \ddot{h}(5)
$$

The non-circulatory components of the unsteady aerodynamic load have been represented in terms of a convolution integral of the indicial Wagner's function $\phi(\tau)$, where the added mass is associated with the term $\frac{1}{2} \rho C_{L \alpha} b^{2} \ddot{h}$.

In order to be able to highlight the implications of the nonlinearity in the delayed control, the structural and aerodynamic models are considered linear. In principle, with the exception of the unavoidable computational expense, the inclusion of these nonlinearities does not constitute a problem. For an aeroelastic model in which structural nonlinearities were included, see e.g. [4]. In addition, in Eq. [4] $L_{b}(\tau)$ denotes the external timedependent load acting on the rigid wing counterpart and $L_{c}(\tau)$ denotes the nonlinear feedback control force:

$$
L_{c}(t)=g_{p} h\left(t-t_{1}\right)+g_{v} \dot{h}\left(t-t_{2}\right)-g_{n c} h^{3}\left(t-t_{3}\right)
$$

In the present work, the proportional (PFC) and velocity (VFC) feedback controls have been supplemented by a 
nonlinear proportional feedback control (with delays $\left.t_{i} ; i=\overline{1,3}\right)$.

Denoting $\tau=t U_{\infty} / b$ and $\xi=h / b$, the governing equation of the system with the nonlinear actuator control force with delay can be written in dimensionless form as

$$
\begin{aligned}
& \underbrace{\xi^{\prime \prime}+2 \zeta_{\xi} \frac{\bar{\omega}}{V} \xi^{\prime}+\left(\frac{\bar{\omega}}{V}\right)^{2} \xi}_{\text {Structural terms }} \\
& =\underbrace{-\frac{2}{\mu} \int_{-\infty}^{\tau} \phi(\tau-\sigma) \xi^{\prime \prime} d \sigma-\frac{1}{\mu} \xi^{\prime \prime}}_{\text {Aerodynamic terms }} \\
& +\underbrace{+g_{p}\left(\frac{\bar{\omega}}{V}\right)^{2} \xi\left(\tau-\tau_{1}\right)+2 \zeta_{\xi} \mathrm{g}_{v} \frac{\bar{\omega}}{V} \xi^{\prime}\left(\tau-\tau_{2}\right)}_{\text {Nonlinear control term }} \\
& -\underbrace{\mathrm{g}_{n c}\left(\frac{\bar{\omega}}{V}\right)^{2} \xi^{3}\left(\tau-\tau_{3}\right)}_{\text {External load }}+\underbrace{L_{b}(\tau)}_{b}
\end{aligned}
$$

The following dimensionless parameters have been used $\mu=m / \pi \rho b^{2} ; V=U_{\infty} / \omega_{0} b ; \bar{\omega}=\omega_{h} / \omega_{0} ; \omega_{h}^{2}=k / m ;$ $\zeta_{\xi}=c / 2 m \omega_{h} ; \mathbf{g}_{v}=g_{v} / c ; \mathbf{g}_{p}=g_{p} / k ; \mathbf{g}_{n c}=g_{n c} b^{2} / k$.

\section{Evaluation of High-Order Aeroelastic Kernels}

Paralleling the procedure presented in [4], assuming a periodic external excitation of the form:

$$
L_{b}(t)=\sum_{j=1}^{n} X_{j} e^{s_{j} t} .
$$

the high order kernels of the aeroelastic system can be derived.

The identification of the $n$ order aeroelastic kernels is based on a general input in the form given by Eq. (8) and on the extraction, for the generic term of $n$-th order, of the coefficients of $\prod_{i=1}^{n} X_{i} e^{s_{i} \tau}$.

This procedure was detailed in [4], where the expressions of the first three Volterra kernels of 2-D lifting surfaces have been explicitly derived.

As a remark, assuming a solution of the plunging displacement in the form $\xi(\tau)=H_{1 \mathrm{OL}}(s) X_{1} e^{s \tau}+\ldots$, the first order kernel, $H_{1 \mathrm{OL}}(s)$ characterizing the open-loop 1-DOF aeroelastic system can be represented as:

$$
H_{1 \mathrm{OL}}(s)=\left\{s^{2}+2 \zeta_{\xi} \frac{\bar{\omega}}{V} s+\left(\frac{\bar{\omega}}{V}\right)^{2}+\frac{2}{\mu}\left[\Phi(s)+\frac{1}{2}\right] s^{2}\right\}^{-1}
$$

In the present case we assume that the feedback control is represented by $\beta(s) ; \beta(s)$ can be one of the $\mathrm{PFC}$, VFC, feedback control gains, or combinations of these (CFC), see [1], in conjunction with a nonlinear proportional control gain.

Based on these assumptions, the linear first Volterra kernel of the closed loop system $H_{1 \mathrm{CL}}(s)$ is given by:

$$
H_{1_{\mathrm{CL}}}(s)=H_{1 \mathrm{OL}}(s) /\left[1+H_{1 \mathrm{OL}}(s) \beta(s)\right]
$$

and is expressed explicitly as

$$
\begin{gathered}
H_{\mathrm{ICL}}(s)=\left\{s^{2}+2 \zeta_{\xi} \frac{\bar{\omega}}{V} s+\left(\frac{\bar{\omega}}{V}\right)^{2}+\frac{2}{\mu}\left[\Phi(s)+\frac{1}{2}\right] s^{2}\right. \\
\left.+\mathrm{g}_{p}\left(\frac{\bar{\omega}}{V}\right)^{2} e^{-s \widetilde{\tau}_{1}}+2 \zeta_{\xi} \mathrm{g}_{v} \frac{\bar{\omega}}{V} s e^{-s \widetilde{\tau}_{2}}\right\}^{-1}
\end{gathered}
$$

where the feedback gains are taken in absolute value. Usually, these gains are negative in the LQG/LQR design methodologies.

For the specific case of the 1-DOF airfoil with linear control $\beta(s)=\mathrm{g}_{p}\left(\frac{\bar{\omega}}{V}\right)^{2} e^{-s \widetilde{\tau}_{1}}+2 \zeta_{\xi} \mathrm{g}_{v} \frac{\bar{\omega}}{V} s e^{-s \widetilde{\tau}_{2}}$.

From (10), when $\beta(s)=0$, i.e. in the case of the openloop, consistently $H_{1 \mathrm{OL}}=H_{1 \mathrm{CL}}$.

From a mathematical point of view, the closed-loop nonlinear aeroservoelastic system, characterized by the first $H_{1 \mathrm{OL}}(s)$ and third $H_{3 \mathrm{OL}}\left(s_{1}, s_{2}, s_{3}\right)$ order kernels and by the feedback gain $\beta(s)$, can be seen as an open loop system described by the closed loop aeroelastic kernels $H_{1 \mathrm{CL}}(s), H_{3 \mathrm{CL}}\left(s_{1}, s_{2}, s_{3}\right)$ that are related to the kernels of the open loop system and its control gains. It should be mentioned that the second order kernel of the actual system is zero by virtue of the fact that no quadratic terms are involved in this system. To obtain the third-order closed loop nonlinear aeroservoelastic kernel $H_{3 \mathrm{CL}}\left(s_{1}, s_{2}, s_{3}\right)$, we assume that the input can be expressed as $L_{b}(\tau)=\sum_{i=1}^{3} X_{i} e^{s_{i} \tau}$. The output $\xi(\tau)$ can be written as:

$$
\begin{aligned}
\xi(\tau) & =\sum_{i=1}^{3} H_{1_{\mathrm{CL}}}\left(s_{i}\right) X_{i} e^{s_{i} \tau}+\sum_{i=1}^{3} H_{1_{\mathrm{CL}}}\left(s_{i}\right) X_{i}^{2} e^{2 s_{i} \tau} \\
& +\sum_{i=1}^{3} H_{\mathrm{l}_{\mathrm{CL}}}\left(s_{i}\right) X_{i}^{3} e^{3 s_{i} \tau} \\
& +3 H_{3_{\mathrm{CL}}}\left(s_{1}, s_{2}, s_{3}\right) X_{1} X_{2} X_{3} e^{\left(s_{1}+s_{2}+s_{3}\right) \tau}+\text { others }
\end{aligned}
$$

Substituting the expression of $\xi(\tau)$ as given by Eq. (13) in Eq. (7) and extracting the $\prod_{i=1}^{3} e^{s_{i} \tau}$ term and using the expression of $H_{\mathrm{l}_{\mathrm{CL}}}\left(s_{i}\right)$, the closed-loop third-order aeroelastic kernel is obtained

$$
\begin{gathered}
H_{3 \mathrm{CL}}\left(s_{1}, s_{2}, s_{3}\right)=2 \mathbf{g}_{n c}\left(\frac{\bar{\omega}}{V}\right)^{2} H_{1 \mathrm{CL}}\left(s_{1}\right) H_{1 \mathrm{CL}}\left(s_{2}\right) H_{1 \mathrm{CL}}\left(s_{3}\right) \\
\times H_{1 \mathrm{CL}}\left(s_{1}+s_{2}+s_{3}\right) e^{-\left(s_{1}+s_{2}+s_{3}\right) \tilde{\tau}}
\end{gathered}
$$

It is a general property of systems that all higher-order kernels can be expressed in terms of the lower-order kernels. In the absence of the quadratic term, see Eq. (7), the second-order kernel vanishes. Therefore, only the first and third order kernels have to be considered toward the determination of the nonlinear aeroelastic response and of the stability boundary.

\section{Stability Analysis}

For stability purposes, the aeroelastic system in the absence of external excitation, $L_{b}(\tau)=0$, is considered. Without aerodynamic terms (that include time-lags), 
and in the absence of control (i.e. $\mathrm{g}_{p}=\mathrm{g}_{v}=0$ ), the system is dissipative with two finite stable characteristic roots (poles) on the left half of the complex plane. However, for the aeroelastic system with feedback delayed control $\left(\tilde{\tau}_{j}>0 ; j=1,2,3\right)$ the two finite stable roots are supplemented by other finite stable roots (whose number depends on the aerodynamic model), and by an additional infinite number of roots due to the presence of $e^{-s \tau}$ into the characteristic equation.

The conditions that guarantee the stability of the delayed system, have been studied by Pontryagin [10], and applied toward the stability of time delayed feedback control systems by several authors. In the present aeroelastic analysis, Pontryagin's approach [10] in conjunction with the Stépán's theorems [13] have been adopted.

As proved in [12], the stability of delayed aeroelastic systems analyzed by using the concept of Retarded Functional Differential Equation (RFDE) depends on the presence of zeros with positive real parts of the characteristic equation, i.e. the presence of the p-zeros. For the stability evaluation, Eq. (11) can be written in characteristic equation form as:

$$
D(s)=1 / H_{1 \mathrm{CL}}(s)=0
$$

As a remark, in the absence of time delay, the following relation is valid

$$
\begin{aligned}
D(s) & =s^{2}+2 \zeta_{\xi} \frac{\bar{\omega}}{V} s+\left(\frac{\bar{\omega}}{V}\right)^{2}+\frac{2}{\mu}\left[\Phi(s)+\frac{1}{2}\right] s^{2} \\
& +\mathrm{g}_{p}\left(\frac{\bar{\omega}}{V}\right)^{2}+2 \zeta_{\xi} \mathbf{g}_{v} \frac{\bar{\omega}}{V} s=0
\end{aligned}
$$

Since $1+\frac{2}{\mu}\left[\Phi(s)+\frac{1}{2}\right]>0$, the stability conditions are obtained by imposing $\mathrm{g}_{v}>-1$ and $\mathrm{g}_{p}>-1$.

Note that the characteristic roots (i.e. poles), of Eq. (15a) are of the form $s=a+i \omega$. As a particular case, if $\mathrm{g}_{p}=\mathrm{g}_{v}=\mathrm{g}$, and $\tilde{\tau}=\tilde{\tau}_{j}$, the following relation holds valid:

$$
\mathbf{g}=\frac{\left|\left\{1+\frac{2}{\mu}\left[\Phi(s)+\frac{1}{2}\right]\right\}\left(\frac{V}{\bar{\omega}}\right)^{2} s^{2}+2 \zeta_{\xi}\left(\frac{V}{\bar{\omega}}\right) s+1\right|}{\left|1+2 \zeta_{\xi} \frac{V}{\bar{\omega}} s\right|} e^{a \tau}
$$

It is possible to observe that for the uncontrolled system, $\mathrm{g}_{p}=\mathrm{g}_{v}=0$, the characteristic equation (15) has four finite stable poles in the complex plane that are obtained by solving the equation

$$
\left|\left\{1+\frac{2}{\mu}\left[\Phi(s)+\frac{1}{2}\right]\right\}\left(\frac{V}{\bar{\omega}}\right)^{2} s^{2}+2 \zeta_{\xi}\left(\frac{V}{\bar{\omega}}\right) s+1\right|=0
$$

and all remaining poles are at $a=-\infty$. For $\mathrm{g}_{p}=\mathrm{g}_{v}=\infty$, there are poles at $s=0 ;-1 ;-82.36 ;-12.89$ and the remaining poles are at $a=+\infty$.

For equal time delays, via time transformation with respect to the delay, i.e. replacing $s \tilde{\tau} \Rightarrow \hat{s}$, Eq. (15) can be further simplified as

$$
\begin{aligned}
D(\hat{s}) & =\hat{s}^{2}+2 \zeta_{\xi} \frac{\bar{\omega}}{V} \hat{s} \tilde{\tau}+\left(\frac{\bar{\omega}}{V}\right)^{2} \tau^{2}+\frac{2}{\mu} \Phi(\hat{s} / \widetilde{\tau}) \hat{s}^{2} \\
& +\frac{1}{\mu} \hat{s}^{2}+g_{p}\left(\frac{\bar{\omega}}{V}\right)^{2} \tilde{\tau}^{2} e^{-\hat{s}}+\mathrm{g}_{v} 2 \zeta_{\xi} \frac{\bar{\omega}}{V} \hat{s} \tilde{\tau} e^{-\hat{s}}=0
\end{aligned}
$$

The stability of Eq. (17) will be studied via Stépán's analytical method $[8,13]$. Following this method, upon denoting $\rho_{1} \geq \ldots \geq \rho_{r} \geq 0$ and $\sigma_{1} \geq \ldots \geq \sigma_{s}=0$, the non-negative real zeros of

$$
R(\omega)=\operatorname{Re} D(i \omega)=(-1)^{m} \omega^{n}+\mathrm{O}\left(\omega^{n}\right)
$$

and

$$
S(\omega)=\operatorname{Im} D(i \omega)=\mathrm{O}\left(\omega^{n}\right),
$$

the trivial solution $x=0$ of the system is exponentially asymptotically stable, if and only if $R(0)>0 ; n=2 m$ ( $n$ is the order of the system and $m$ is integer); $S\left(\rho_{k}\right) \neq 0$ for $k=1, \ldots, r$ and

$$
\sum_{k=1}^{r}(-1)^{k} \operatorname{sgn} S\left(\rho_{k}\right)=(-1)^{m} m
$$

Similar conditions of stability are defined for systems where $n=2 m+1$, see [8]. For the present case, replacing $\Phi(\hat{s}) \hat{s}^{2} \Rightarrow C(\hat{s}) \hat{s} \widetilde{\tau}$ and $\hat{s} \Rightarrow i \omega$, where $C(\bar{k})$ $(\equiv F(\bar{k})+i G(\bar{k}))$ is the Theodorsen's function, and considering the real and imaginary parts of Eq. (17), we obtain:

$$
\begin{gathered}
R(\omega)=-\omega^{2}+\left(\frac{\bar{\omega}}{V}\right)^{2} \tilde{\tau}^{2}-\frac{2}{\mu} \omega \tilde{\tau} G(\bar{k} / \tilde{\tau}) \omega-\frac{1}{\mu} \omega^{2} \\
+\mathrm{g}_{p}\left(\frac{\bar{\omega}}{V}\right)^{2} \tilde{\tau}^{2} \cos \omega+2 \zeta_{\xi} \frac{\bar{\omega}}{V} \mathrm{~g}_{v} \tilde{\tau} \omega \sin \omega \\
S(\omega)=2 \zeta_{\xi} \frac{\bar{\omega}}{V} \tilde{\tau} \omega+\frac{2}{\mu} \omega \tilde{\tau} F(\bar{k} / \tilde{\tau}) \\
\quad-\mathrm{g}_{p}\left(\frac{\bar{\omega}}{V}\right)^{2} \tilde{\tau}^{2} \sin \omega+2 \zeta_{\xi} \frac{\bar{\omega}}{V} \mathrm{~g}_{v} \tilde{\tau} \omega \cos \omega
\end{gathered}
$$

The trivial solution of Eq. (7) is exponentially asymptotically stable $[8,23]$, if, and only if:

$$
\begin{gathered}
\mathrm{g}_{p}>-1 \\
\tilde{\tau}<2 \zeta_{\xi} \frac{V}{\bar{\omega}} \frac{\mathrm{g}_{v}}{\mathrm{~g}_{p}} \\
\mathrm{~g}_{p}<\frac{\sigma}{\tau}\left(\frac{V}{\bar{\omega}}\right)^{2}\left\{\frac{\sigma}{\tau}-\left(\frac{\bar{\omega}}{V}\right)^{2} \frac{\tilde{\tau}}{\sigma}+\frac{1}{\mu} \frac{\sigma}{\tau}\right] \cos \sigma+2 \zeta_{\xi} \frac{\bar{\omega}}{V} \sin \sigma \\
\left.+\frac{2}{\mu}(G \cos \sigma+F \sin \sigma)\right\}
\end{gathered}
$$

Herein, $\sigma$ is the smallest positive zero of the equation

$$
\begin{aligned}
S(\sigma) & =2 \zeta_{\xi} \frac{\bar{\omega}}{V} \tilde{\tau} \sigma+\frac{2}{\mu} \sigma \tilde{\tau} F \\
& -\mathrm{g}_{p}\left(\frac{\bar{\omega}}{V}\right)^{2} \tau^{2} \sin \sigma+2 \zeta_{\xi} \frac{\bar{\omega}}{V} \mathrm{~g}_{v} \tilde{\tau} \sigma \cos \sigma=0
\end{aligned}
$$

where $\sigma \in\left(0, \frac{\pi}{2}\right)$. The proof of Eq. (17) is given next.

The inequality $\mathrm{g}_{p}>-1$ is obtained from the condition:

$$
R(0)=\left(\frac{\bar{\omega}}{V}\right)^{2} \widetilde{\tau}^{2}+\mathrm{g}_{p}\left(\frac{\bar{\omega}}{V}\right)^{2} \widetilde{\tau}^{2}>0
$$

Considering the smallest positive root $\sigma$ of Eq. (20b), one obtains that, $S(\omega)>0, \omega \in(0, \sigma)$ if and only if 
44th AIAA/ASME/ASCE/ASC Structures, Structural Dynamics, and Materials Conference, Norfolk, VA, 7-10 April 2003

$$
\begin{aligned}
2 \zeta_{\xi} \frac{\bar{\omega}}{V} \omega & +\frac{2}{\mu} \omega F-\mathrm{g}_{p}\left(\frac{\bar{\omega}}{V}\right)^{2} \tau \sin \omega \\
& +2 \zeta_{\xi} \frac{\bar{\omega}}{V} \mathrm{~g}_{v} \omega \cos \omega>0
\end{aligned}
$$

that yields

$$
\mathbf{g}_{p}\left(\frac{\bar{\omega}}{V}\right)^{2} \tau \sin \omega<2 \zeta_{\xi} \frac{\bar{\omega}}{V} \omega+\frac{2}{\mu} \omega F+2 \zeta_{\xi} \frac{\bar{\omega}}{V} \mathbf{g}_{v} \tau \omega \cos \omega
$$

Since the first two terms of Eq. (25) are always positive, it is possible to conclude that the condition $\tilde{\tau}<2 \zeta_{\xi} \frac{V}{\bar{\omega}} \frac{\mathrm{g}_{v}}{\mathrm{~g}_{p}}$ is required. In addition, for the smallest positive root $\sigma$, we can state:

$$
\begin{aligned}
R(\sigma) & =-\sigma^{2}+\left(\frac{\bar{\omega}}{V}\right)^{2} \tilde{\tau}^{2}-\frac{2}{\mu} \sigma \tilde{\tau} G \sigma-\frac{1}{\mu} \sigma^{2} \\
& +\mathrm{g}_{p}\left(\frac{\bar{\omega}}{V}\right)^{2} \widetilde{\tau}^{2} \cos \sigma+2 \zeta_{\xi} \frac{\bar{\omega}}{V} \mathrm{~g}_{v} \tilde{\tau} \sigma \sin \sigma<0
\end{aligned}
$$

After some algebra manipulations

$$
\begin{gathered}
\mathrm{g}_{p} \cos ^{2} \sigma<\left[\left(\frac{V}{\bar{\sigma}} \frac{\sigma}{\widetilde{\tau}}\right)^{2}-1+\frac{2}{\mu} \frac{\sigma}{\widetilde{\tau}}\left(\frac{V}{\bar{\omega}}\right)^{2} G-\frac{1}{\mu}\left(\frac{V}{\bar{\omega}} \frac{\sigma}{\widetilde{\tau}}\right)^{2}\right. \\
\left.-2 \zeta_{\xi}\left(\frac{V}{\bar{\omega}} \frac{\sigma}{\tilde{\tau}}\right) \mathrm{g}_{v} \sin \sigma\right] \cos \sigma
\end{gathered}
$$

and using standard trigonometric relationships, Eq. (26b) in conjunction with Eq. (25), rewritten in the form

$$
\begin{gathered}
\mathrm{g}_{p} \sin ^{2} \sigma<\left[2 \zeta_{\xi}\left(\frac{V}{\bar{\omega}} \frac{\sigma}{\tilde{\tau}}\right)+\frac{2}{\mu} \frac{\sigma}{\tau}\left(\frac{V}{\bar{\sigma}}\right)^{2} F\right. \\
\left.+2 \zeta_{\xi}\left(\frac{V}{\bar{\omega}} \frac{\sigma}{\tau}\right) g_{v} \sigma \cos \sigma\right] \sin \sigma
\end{gathered}
$$

reduces to Eq. (21c). The condition (19), where $m=1$, is also fulfilled.

\section{The Aeroelastic Stability Chart}

The present approach for the determination of the stability domain of a delayed aeroelastic system has some analogies with Theodorsen's method used for finding the flutter speed by plotting the real and imaginary parts of the flutter determinant in conjunction with consideration of a real $\omega$. The former approach reduces to the latter one in the case of zero time delays. The stability chart of the aeroelastic system described in Eq. (7) with respect to the feedback gains and the timedelay can be constructed using Stépán's theorem and the D-subdivision method [12]. From the preliminary findings it appears that the time-delays play an important role. It is noted that the stability boundary depends dramatically on the velocity feedback control, especially in the case of the time delay. In addition, this implies that, in the presence of the delays, a small variation in the velocity feedback gain can expel the system from a stable to an unstable domain.

\section{HOPF-BIFURCATION ANALYSIS}

Preliminary findings related to the Hopf-bifurcation analysis of nonlinear feedback time delayed closed-loop aeroelastic systems will be presented next.

Based on $[8,24]$, assuming for sake of simplicity equal time-delays $\tau_{1}=\tau_{2}=\tau_{3}=\tilde{\tau}$, expanding the nonlinear time-delayed feedback control $L_{c}$ into Taylor series and omitting the terms containing the nonlinear function of the time-delay yields:

$$
\begin{aligned}
L_{c}(t)= & -g_{v} \tilde{\tau} \ddot{h}(t)+\left(g_{v}-g_{p} \tilde{\tau}\right) \dot{h}(t)+g_{p} h(t) \\
& +g_{n c} h^{3}(t)-3 g_{n c} \widetilde{\tau} h^{2}(t) \dot{h}(t)+\mathrm{O}\left(\widetilde{\tau}^{2}\right)
\end{aligned}
$$

As a result, the aeroelastic governing equation is rewritten in the form

$$
\begin{aligned}
& m \ddot{h}(t)+c \dot{h}(t)+k h(t)+g_{v} \ddot{\tau} \ddot{h}(t)-\left(g_{v}-g_{p} \tilde{\tau}\right) \dot{h}(t)-g_{p} h(t) \\
& -g_{n c} h^{3}(t)+3 g_{n c} \widetilde{\tau} h^{2}(t) \dot{h}(t)=-C_{L \alpha} \rho b U_{\infty} \int_{-\infty}^{t} \phi(t-\sigma) \ddot{h}(t) d \sigma \\
& -\frac{1}{2} \rho C_{L \alpha} b^{2} \ddot{h}(t)+L_{c}(t)
\end{aligned}
$$

and in dimensionless form as

$$
\begin{aligned}
& \xi^{\prime \prime}+2 \zeta_{\xi} \frac{\bar{\omega}}{V} \xi^{\prime}+\left(\frac{\bar{\omega}}{V}\right)^{2} \xi=-\frac{2}{\mu} \int_{-\infty}^{\tau} \phi(\tau-\sigma) \xi^{\prime \prime} d \sigma-\frac{1}{\mu} \xi^{\prime \prime} \\
& -2 \zeta_{\xi} \mathrm{g}_{v} \frac{\bar{\omega}}{V} \widetilde{\tau} \xi^{\prime \prime}+\left[2 \zeta_{\xi} \mathrm{g}_{v} \frac{\bar{\omega}}{V}-\mathrm{g}_{p}\left(\frac{\bar{\omega}}{V}\right)^{2} \tau\right] \xi^{\prime}+\mathrm{g}_{p}\left(\frac{\bar{\omega}}{V}\right)^{2} \xi \\
& +\mathrm{g}_{n c}\left(\frac{\bar{\omega}}{V}\right)^{2} \xi^{3}-3 \mathrm{~g}_{n c}\left(\frac{\bar{\omega}}{V}\right)^{2} \widetilde{\tau} \xi^{2} \xi^{\prime}
\end{aligned}
$$

Usually the gains are negative in the LQR design, and so are taken in absolute value. This yields the following approximated nonlinear equation:

$$
\begin{aligned}
& \left(1-2 \zeta_{\xi} \mathbf{g}_{v} \frac{\bar{\omega}}{V} \widetilde{\tau}-\frac{1}{\mu}\right) \xi^{\prime \prime}+\left[2 \zeta_{\xi} \frac{\bar{\omega}}{V}+2 \zeta_{\xi} \mathrm{g}_{v} \frac{\bar{\omega}}{V}-\mathrm{g}_{p}\left(\frac{\bar{\omega}}{V}\right)^{2} \tau \mid \xi^{\prime}\right. \\
& +\left(\frac{\bar{\omega}}{V}\right)^{2}\left(1+\mathrm{g}_{p}\right) \xi+\mathrm{g}_{n c}\left(\frac{\bar{\omega}}{V}\right)^{2}\left(\xi^{3}-3 \widetilde{\tau} \xi^{2} \xi^{\prime}\right) \\
& +\frac{2}{\mu} \int_{-\infty}^{\tau} \phi(\tau-\sigma) \xi^{\prime \prime} d \sigma=0
\end{aligned}
$$

\section{$\underline{\text { Stability examination }}$}

The zero solution of Eq. (31), for the system in vacuum, is exponentially asymptotically stable $[8,24]$ if

$$
\begin{gathered}
\mathrm{g}_{p}>-1 \\
1-2 \zeta_{\xi} \mathrm{g}_{v} \frac{\bar{\omega}}{V} \tilde{\tau}>0 ; \\
2 \zeta_{\xi} \frac{\bar{\omega}}{V}+2 \zeta_{\xi} \mathrm{g}_{v} \frac{\bar{\omega}}{V}-\mathrm{g}_{p}\left(\frac{\bar{\omega}}{V}\right)^{2} \tau>0
\end{gathered}
$$

The Hopf-Bifurcation (HB) is present at

$$
\frac{\mathrm{g}_{p}}{\left(1+\mathrm{g}_{v}\right)}=\frac{2 \zeta_{\xi}}{\frac{\bar{\omega}}{V} \widetilde{\tau}} ; \mathrm{g}_{v}<\frac{1}{2 \zeta_{\xi} \frac{\bar{\omega}}{V} \widetilde{\tau}} ; \mathrm{g}_{p}>-1
$$

and it is supercritical or subcritical if $g_{n c}<0$ or $\mathrm{g}_{n c}>0$, respectively. The proof, paralleling that presented in [8] is given next.

The nonlinear Eq. (31) can be linearized about $\xi=0$

$$
\begin{aligned}
& \left(1-2 \zeta_{\xi} \mathrm{g}_{v} \frac{\bar{\omega}}{V} \tau\right) \xi^{\prime \prime}+\left\lfloor 2 \zeta_{\xi} \frac{\bar{\omega}}{V}+2 \zeta_{\xi} \mathrm{g}_{v} \frac{\bar{\omega}}{V}-\mathrm{g}_{p}\left(\frac{\bar{\omega}}{V}\right)^{2} \tau\right] \xi^{\prime} \\
& +\left(\frac{\bar{\omega}}{V}\right)^{2}\left(1+\mathrm{g}_{p}\right) \xi=0
\end{aligned}
$$

and its characteristic polynomial is given by

$$
\begin{aligned}
& \left(1-2 \zeta_{\xi} \mathbf{g}_{v} \frac{\bar{\omega}}{V} \tilde{\tau}\right) s^{2}+\left[2 \zeta_{\xi} \frac{\bar{\omega}}{V}+2 \zeta_{\xi} \mathbf{g}_{v} \frac{\bar{\omega}}{V}-\mathbf{g}_{p}\left(\frac{\bar{\omega}}{V}\right)^{2} \tau\right] s \\
& +\left(\frac{\bar{\omega}}{V}\right)^{2}\left(1+\mathrm{g}_{p}\right)=0
\end{aligned}
$$

Assuming that $\mathrm{g}_{v}<\frac{V}{2 \zeta_{\xi} \overline{\boldsymbol{\omega}} \tilde{\tau}}$ and $\mathrm{g}_{v}=\frac{\bar{\omega} \tilde{\tau}}{2 \zeta_{\xi} V} \mathbf{g}_{p}-1$, then, as a necessary requirement for the Hopf-bifurcation, we 
44th AIAA/ASME/ASCE/ASC Structures, Structural Dynamics, and Materials Conference, Norfolk, VA, 7-10 April 2003

have the condition of a pair of pure imaginary roots of the characteristic equation

$$
s_{1,2}= \pm i \beta \text { where } \beta=\sqrt{\frac{1+\mathrm{g}_{p}}{\left(\frac{V}{\bar{\omega}}\right)^{2}+2 \zeta_{\xi} \frac{V}{\bar{\omega}} \tilde{\tau}-\tilde{\tau}^{2} \mathrm{~g}_{p}}}
$$

If $V=0$ then $\beta=\sqrt{\frac{1+\mathrm{g}_{p}}{-\tilde{\tau}^{2} \mathrm{~g}_{p}}}$.

Upon defining the new variables $y_{1}=\xi$ and $y_{2}=\xi^{\prime} / \beta$ the Poincaré normal form is obtained:

$$
\left[\begin{array}{l}
y_{1}^{\prime} \\
y_{2}^{\prime}
\end{array}\right]=\left[\begin{array}{cc}
0 & -\beta \\
-\beta & 0
\end{array}\right]\left[\begin{array}{l}
y_{1} \\
y_{2}
\end{array}\right]+\left[\begin{array}{c}
0 \\
f_{30} y_{1}^{3}+f_{21} y_{1}^{2} y_{2}
\end{array}\right]
$$

where

$$
\begin{aligned}
& f_{30}=-\frac{1}{\beta^{3}}\left(1+\mathrm{g}_{p}\right) \mathrm{g}_{n c} \\
& f_{21}=\frac{3 \tilde{\tau}}{\beta^{2}}\left(1+\mathrm{g}_{p}\right) \mathrm{g}_{n c}=-3 \tilde{\tau} \beta f_{30}
\end{aligned}
$$

The type of HB occurring at the critical time-delay can be determined by using the center manifold theorem. Specifically, the sign of the quantity $L$, where

$$
\mathrm{L}=\frac{1}{8} f_{21}
$$

determined at the critical time-delay, defines the benign flutter boundary (supercritical HB) and the catastrophic one (subcritical HB), for the cases $L<0$ and $L>0$, respectively. This implies that

$$
\begin{aligned}
& f_{21}<0 \Rightarrow \mathrm{g}_{n c}<0, \text { supercritical HB } \\
& f_{21}>0 \Rightarrow \mathrm{g}_{n c}>0, \text { subcritical HB } \\
& \mathrm{g}_{n c}=0 \text { degenerated HB. }
\end{aligned}
$$

\section{RESULTS AND DISCUSSION}

The parameters for the simulation are presented in Table 1

Table 1. Airfoils and flow parameters

\section{1-DOF Plunging Airfoil}

\begin{tabular}{cc}
$b=1 \mathrm{ft}$ & $\rho_{\infty}=0.0318 \mathrm{slugs} / \mathrm{ft}^{3}$ \\
$\zeta=0.008$ & $c=2 m \omega \zeta$ \\
$m=10 \mathrm{slugs} / \mathrm{ft}$ & $k=\omega^{2} m$ \\
$\omega_{h}=60 \mathrm{rad} / \mathrm{s}$ & $C_{L \alpha}=2 \pi$ \\
\hline \hline
\end{tabular}

In Fig. 1 the effect of the time delay on the first order kernel is presented for selected values of the proportional and velocity feedback gains and for selected values of the dimensionless frequency $\omega / \omega_{h}$, respectively. In Figs. 2 and 3, 3-D and contour plots of the first and third order kernels with the variation of the frequency and of the time delay are depicted for two values of the proportional and velocity feedback gains and two values of the time delay, respectively.

The stability chart of the aeroelastic system described by Eq. (7) with respect to the feedback gains and the time-delay is constructed using the second Stépán's second theorem and the D-subdivision method.
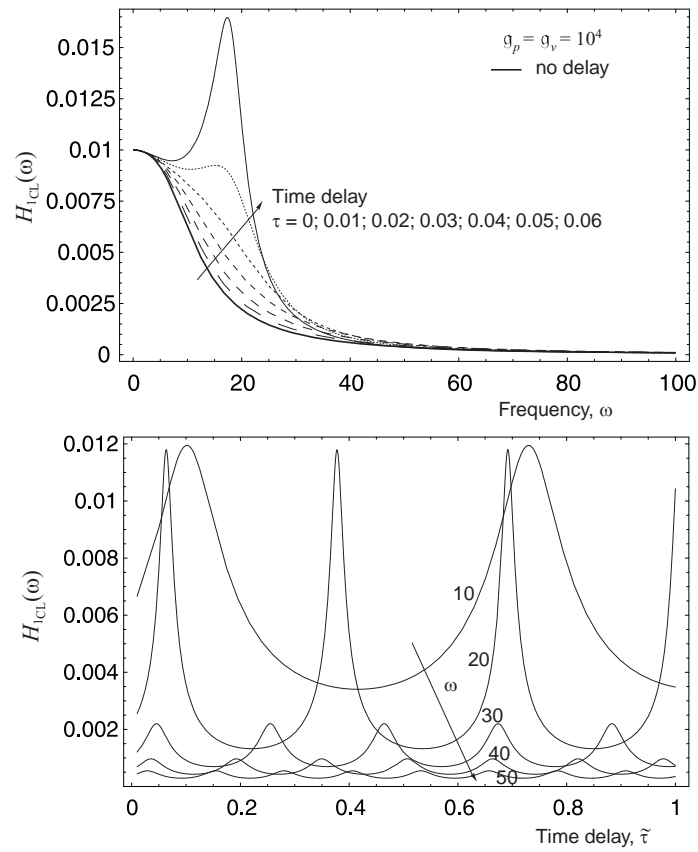

Fig. 1 First order aeroelastic kernel. Effect of the time delay. a) $H_{l} v s$. $\omega$. Influence of the time delay; b) $H_{l} v s$. $\tau$. Influence of the frequency.

The method of D-subdivision is applied for determining the condition under which the quasi-polynomial $D_{c}(s)$ has no p-zeros.

As remarked in Ref. [3], since the quasi-polynomial is a continuous function of its parameters we can construct the subdivision of the coefficient's space by hypersurfaces, the points of which are quasi-polynomials with at least one imaginary root. In addition, as it has been proven in [12], with the variation of the quasipolynomial parameters the number of p-zeros may change only by passage of some zeros through an imaginary axis, and that for all points of every domain of D-subdivision the number of quasi-polynomial $\mathrm{p}$ zeros will be the same.

The region in the $\left\{g_{p}, g_{v}\right\}$ parameter space where the roots of the characteristic equation of the system have zero real part has been represented in Fig. 4 in which the values of the geometrical parameters have been locked. Other stability regions can be drawn in the $\left\{g_{p}, g_{v}\right\}$ parameter space, but these are not of practical importance. As clearly presented, the time-delays play an important role. It is noted that the range of stability for $\mathrm{g}_{p}$ is much larger than that of $\mathrm{g}_{v}$, implying that the stability boundary depends dramatically on the velocity feedback gain, especially in the case of the time delay. 
44th AIAA/ASME/ASCE/ASC Structures, Structural Dynamics, and Materials Conference, Norfolk, VA, 7-10 April 2003

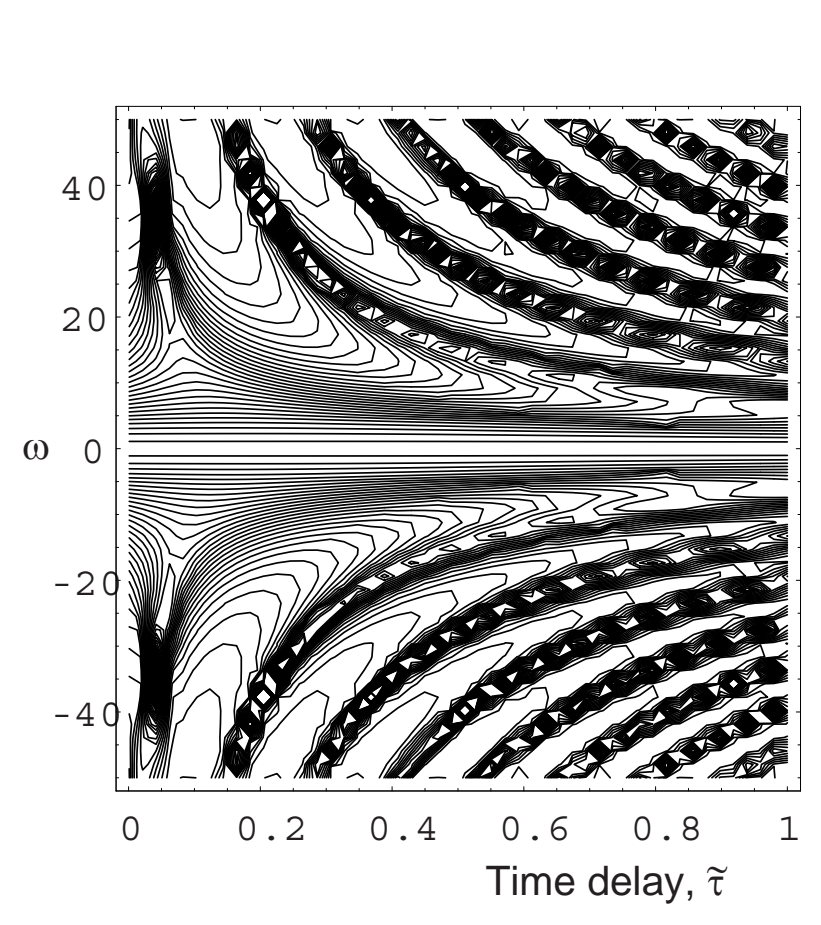

$$
g_{p}=g_{v}=1.0 \cdot 10^{4}
$$
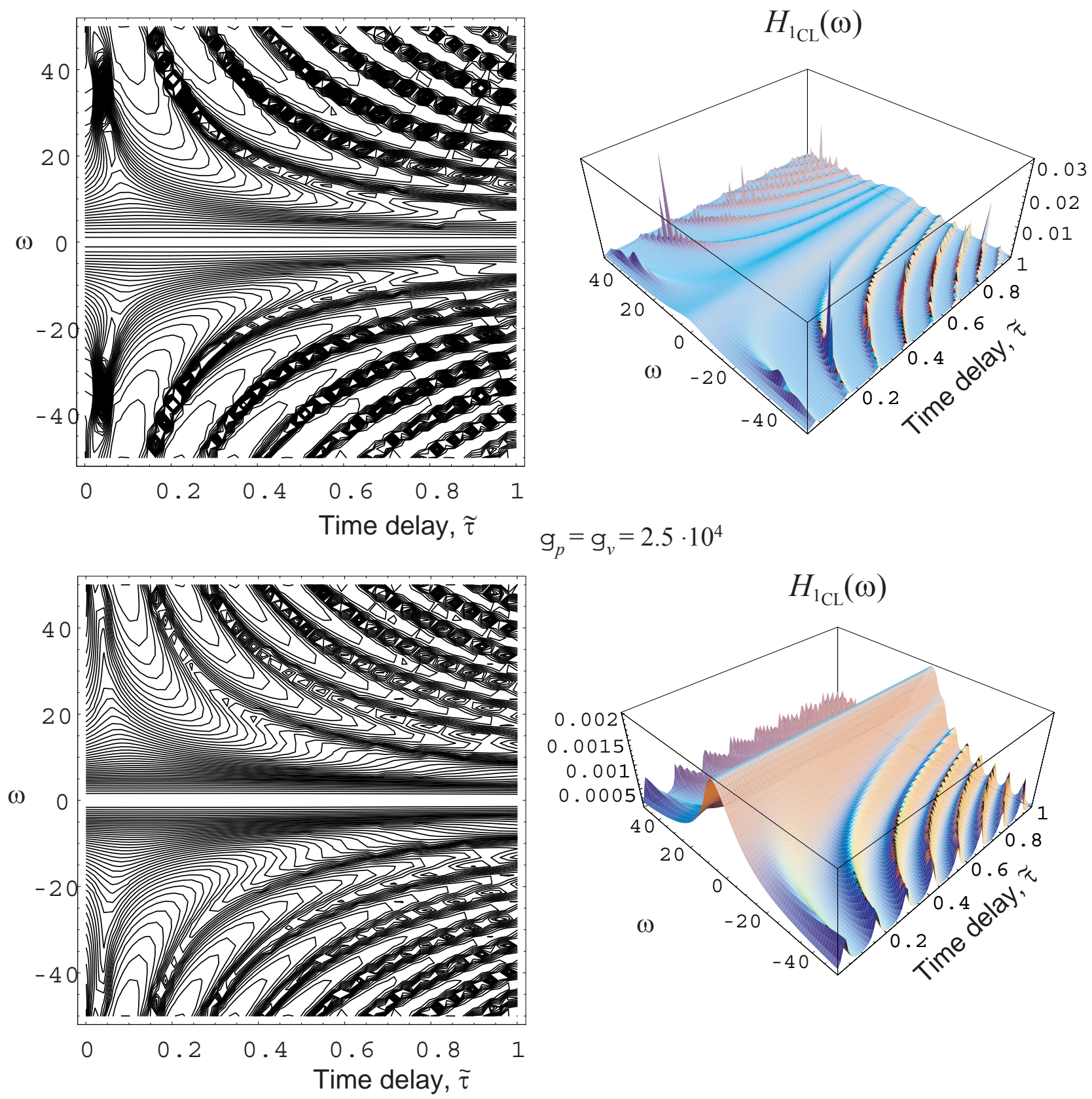

$$
g_{p}=g_{v}=2.5 \cdot 10^{4}
$$

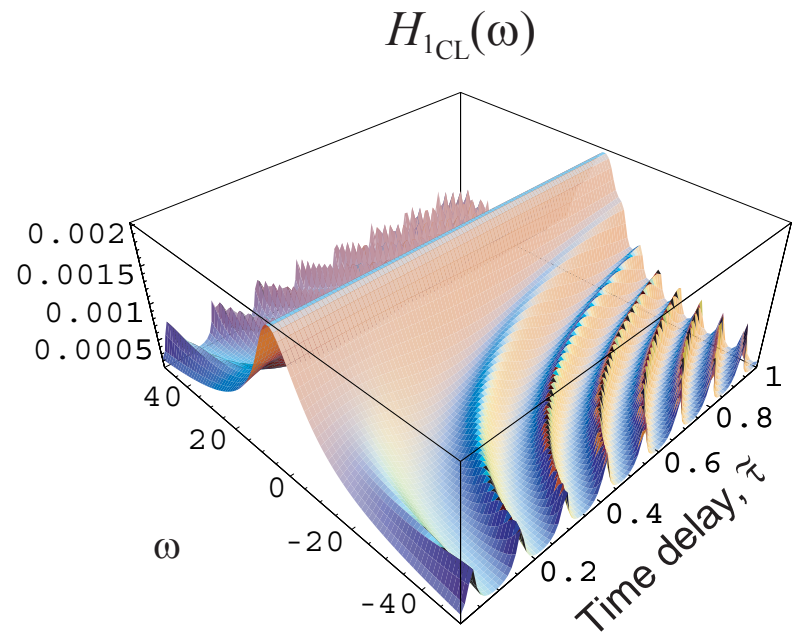

Fig. 2 3-D and contour plots of the first order kernel. Variation of the frequency vs. time delay for two values of the proportional and velocity feedback gains. 
44th AIAA/ASME/ASCE/ASC Structures, Structural Dynamics, and Materials Conference, Norfolk, VA, 7-10 April 2003
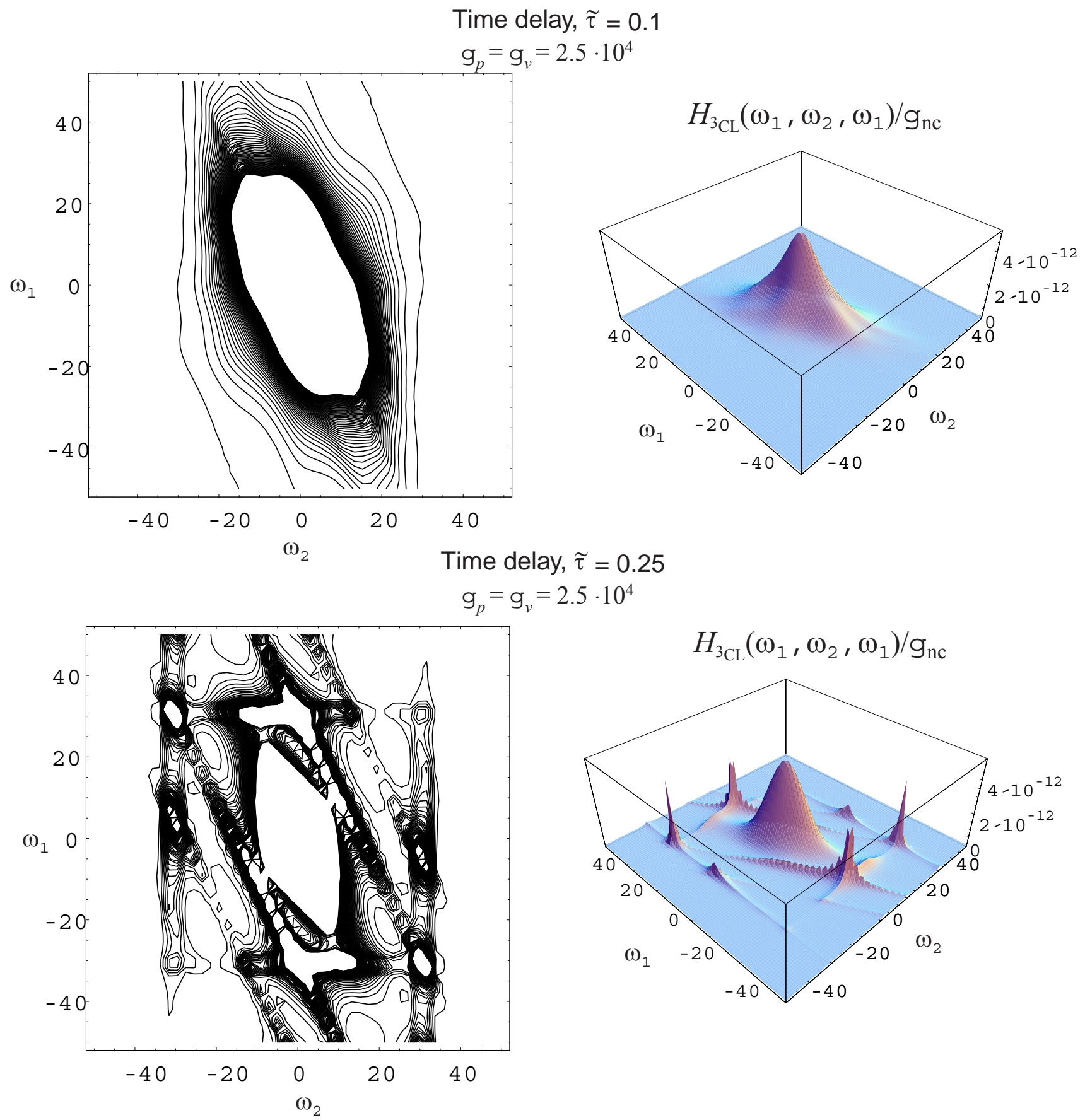

Fig. 3 3-D and contour plots of the third order kernel. Variation of the frequency vs. the time delay for two values of the time delay. 
44th AIAA/ASME/ASCE/ASC Structures, Structural Dynamics, and Materials Conference, Norfolk, VA, 7-10 April 2003

In addition this implies that, in the presence of the delays, a small variation in the velocity feedback gain can expel the system from the stable domain to the unstable domain. On the other hand, the stable parameter space is the complete positive quadrant of $\left\{g_{p}, g_{v}\right\}$ parameter plane if no time delays are present, independent of the values of the feedback gains.

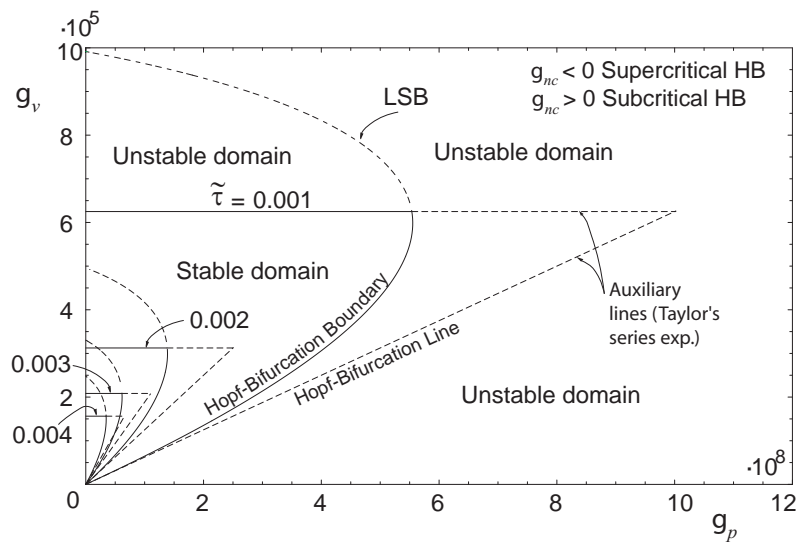

Fig. 4 Stability charts of the full and approximate (Taylor's expansion) closed-loop delayed nonlinear aeroelastic system.

Whereas the linear analyses are able to predict the stability boundary, the nonlinear analyses provide an insight on the character of the stability boundary. To this purpose an auxiliary stability chart (see Fig. 4), corresponding to the statement of Eqs. (33), enables the prediction of the type of Hopf-Bifurcation (i.e. supercritical or subcritical HB). This auxiliary plot for small time-delay, supplies full information related to the HB but, due to the simplicity of the Taylor's series expansion used, provides other types of instability. For this reason the auxiliary plot is used only to determine the HB conditions and not the analytical examination of the full nonlinear system.

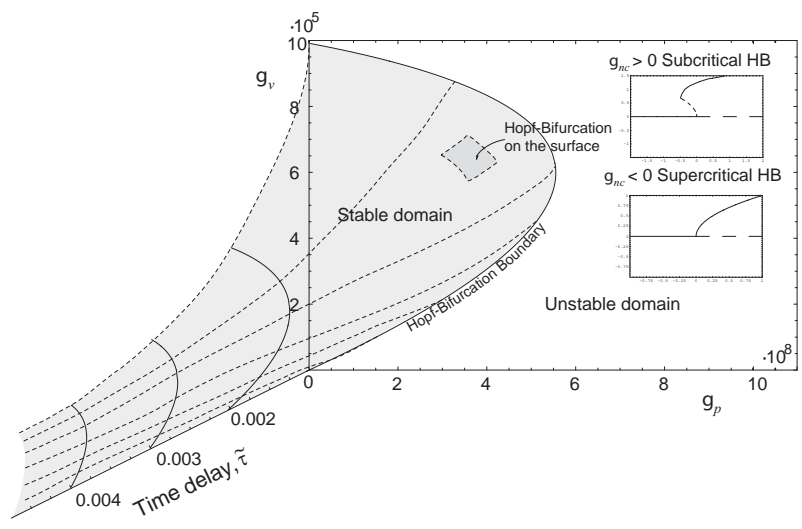

Fig. 5 3-D stability chart of the plunging airfoil. Effect of the time delay and of the control gains.
On the basis of the HB condition and of Fig. 4, it can be seen that the HB occurs on the 3-D surface of the parametric domain depicted in Fig. 5. Therefore, if the parameter point is situated in the inner domain, the system is stable, and if the parameter point is in the outer domain, the system reaches a stable or unstable LCO if the nonlinear control gain is negative or positive, respectively. In Fig. 6 the nonlinear closedloop aeroelastic responses of the 1-DOF airfoil for $\mathrm{g}_{n c}=-10$ (implying a stable LCO, i.e a supercritical HB) and $g_{n c}=10$ (implying an unstable LCO, i.e. a subcritical HB) are presented. These results, obtained via both Volterra series and numerical integration, are in agreement with the analytical predictions of Eqs. $(40 \mathrm{a}, \mathrm{b})$.
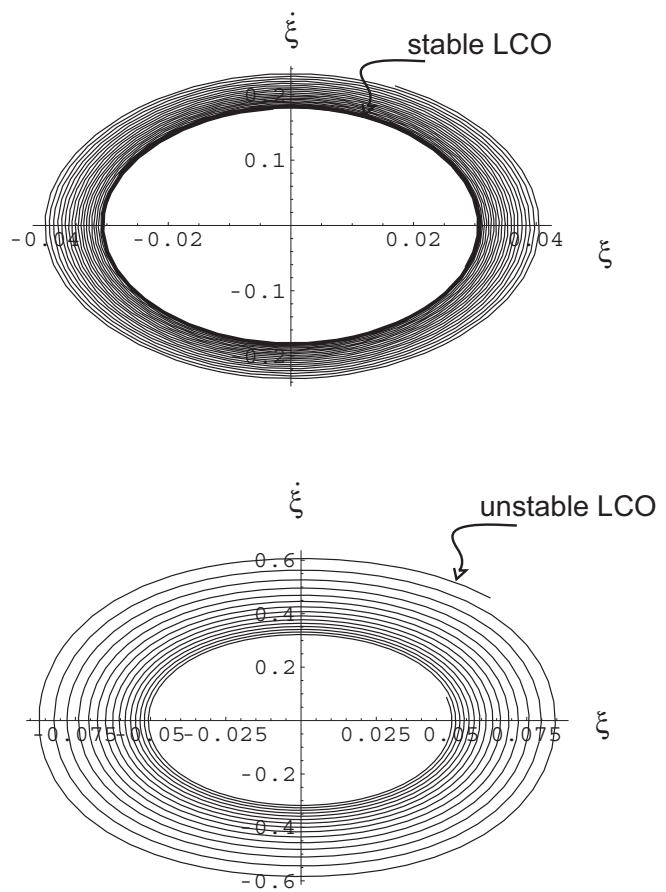

Fig. 6 Stable and unstable LCO for the plunging airfoil with active feedback delayed control law $(\tilde{\tau}=0.01)$.

\section{CONCLUSIONS}

This paper constitutes a basic step towards a better understanding of a number of issues related to the nonlinear aeroservoelasticity of 2-D and 3-D aircraft wings and their nonlinear feedback control featuring time-delays. The model presented here, based on the determination of higher order Volterra kernels, can be used toward the determination of the nonlinear aeroelastic response and flutter boundary of open/closed-loop aeroelastic systems. The effects of the control parameters and of the delay in the state feedback on the flutter boundary and on the stable/unstable LCO have been highlighted. The importance of the time delay 
44th AIAA/ASME/ASCE/ASC Structures, Structural Dynamics, and Materials Conference, Norfolk, VA, 7-10 April 2003

on the nonlinear feedback control has been emphasized. With the incorporation of structural and aerodynamic nonlinearities in the system, or even in the feedback loop, more complex phenomena (LCO and chaos) are expected to occur.

\section{ACKNOWLEDGMENT}

The partial support of this research by the NASA Langley Research Center through Grant NAG-1-02-011 is gratefully acknowledged.

\section{REFERENCES}

1. Marzocca, P., Librescu, L., and Silva, W. A., "Open/Closed-Loop Nonlinear Aeroelasticity for Airfoils via Volterra Series Approach" AIAA Paper 2002-1484, 43rd AIAA/ASME/ASCE/AHS/ASC Structures, Structural Dynamics, and Materials Conference, April 22-25, 2002, Denver, CO.

2. Hu, H. Y., Dowell, E. H., and Virgin, L. N., "Stability Estimation of High Dimensional Vibrating Systems Under State Delay Feedback Control," Journal of Sound and Vibration, Vol. 214, No. 3, 1998, pp. 497-511.

3. Librescu, L., Marzocca, P., and Silva, W. A., "Time Delay Feedback Aeroelastic Control of 2-D Lifting Surfaces," IMECE 2002-32971, 5th International Symposium on Fluid-Structure Interaction, Aeroelasticity, Flow-Induced Vibration \& Noise 2002 ASME Int'l Mechanical Engineering Congress \& Exposition, 17-22 November 2002, New Orleans, Louisiana.

4. Marzocca, P., Librescu, L., and Silva, W. A., "Aeroelastic Response of Nonlinear Wing Section by Functional Series Technique," AIAA Journal, Vol. 40, No. 5, 2002, pp. 813-824.

5. Ott, E. Grebogi, C., and Yorke, J. A., "Controlling Chaos," Physical Review Letters, Vol. 64, 1990, pp. 1196-1199.

6. Ramesh, M., and Narayanan, S., "Controlling Chaotic Motions in a Two-Dimensional Airfoil Using Time-Delayed Feedback," Journal of Sound and Vibration, Vol. 239, No. 5, 2001, pp. 10371049.

7. Pyragas, K., "Continuous Control of Chaos by SelfControlling Feedback," Physics Letters A, Vol. 170, 1992, pp. 421-428.

8. Palkovics, L., and Venhovens, P. J. Th., "Investigation on Stability and possible Chaotic Motions in the Controlled Wheel Suspensions Systems," Vehicle System Dynamics, Vol. 21, 1992, pp. 269-296.

9. Myshkis, A. D., "General Theory of Differential Equations with Delay," American Mathematical Society Translations, Vo. 55, 1951, pp. 1-62.

10. Pontrjagin, L. S., "On the Zeros of Some Elementary Transcendental Functions," American
Mathematical Society Translations, Vol. 2, No. 1, 1955, pp. 95-110.

11. Górecki, H., and Popek, L., "Control of the Systems with Time Delay," IFAC 3rd Symposium Control of Distributed Parameter Systems, 1982, Toulouse, France.

12. Kolmanovskii, V. B., and Nosov, V. R., Stability of Functional Differential Equations, Vol. 180, Mathematics in Science and Engineering, Academic Press, Inc., London, UK, 1986.

13. Stépán, G., Retarded Dynamical Systems: Stability and Characteristic Functions, $\pi$ Pitman Research Notes in Mathematics Series, Vol. 210, Longman Scientific and Technical, co-published in the USA with John Wiley \& Sons, New York, 1989.

14. Marshall, J. E., Górecki, Walton, K., Korytowski, A., Time-Delay Systems, Stability and Performance Criteria with Applications, G.M. Bell Editor, Series in Mathematics and its Applications, Ellis Horwood Ltd, Chichester, West Sussex, England, 1992.

15. Olgac, N., and Holm-Hansen, B. T., "A Novel Active Vibration Absorption Technique: Delayed Resonator," Journal of Sound and Vibration, Vol. 176, No. 1, 1994, pp. 93-104.

16. Just, W., Bernard, T., Ostheimer, M., Reibold, E. and Benner; H., "On the Mechanism of TimeDelayed Feedback Control," Physical Review Letters, Vol. 78, 1997, pp. 203-206.

17. Hu, H. Y., and Wang, Z. H., "Stability Analysis of Damped SDOF Systems with Two Time Delays in State Feedback," Journal of Sound and Vibration, Vol. 214, No. 2, 1998, pp. 213-225.

18. Volterra, V., Theory of Functionals and of Integral and Integro-Differential Equations, Dover Publications, Inc., New York, 1959.

19. Rugh, W. J., Nonlinear Systems Theory, The Volterra-Wiener Approach, The Johns Hopkins University Press, 1981.

20. Worden, K., and Tomlinson, G.R., Nonlinearity in Structural Dynamics Detection, Identification and Modelling, IoP, Institute of Physics Publishing Ltd, Bristol, 2001.

21. Özbay, H., Bachmann, G. R., “ $H^{2} / H^{\infty}$ Controller Design for a Two-Dimensional Thin Airfoil Flutter Suppression," Journal of Guidance, Control, and Dynamics, Vol. 17, No. 4, 1994, pp. 722-728.

22. Scanlan, R. H., Rosenbaum, R., Introduction to the Study of Aircraft Vibration and Flutter, Macmillan, New York, 1951.

23. Ko, J., Kurdila, A.J., and Strganac, T.W., "Nonlinear Control Theory for a Class of Structural Nonlinearites in a Prototypical Wing Section," Journal of Guidance, Control, and Dynamics, Vol. 20, No. 6, 1997, pp. 1181-1189.

24. Guckenheimer, J., Holmes, P., Nonlinear Oscillations, Dynamical Systems, and Bifurcations of Vector Field. New York, Springer, 1986. 